\title{
Defect structure in nanoalloys
}

\author{
Miguel José-Yacamán, ${ }^{* a}$ Eduardo Pérez-Tijerina $^{b}$ and Sergio Mejía-Rosales ${ }^{b}$
}

DOI: $10.1039 / \mathrm{b} 617965 \mathrm{~g}$

The defect structure of bimetallic nanoparticles differs from that of particles made of a single atomic species. Using high resolution TEM imaging along with molecular dynamics simulations, it is possible to investigate the nature and features of these defects. The definition of a local order parameter allows one to locate regions with different kinds of stacking on the simulated nanoparticles and thus to make a direct comparison with the experimental observations.

In recent times there has been much interest in the understanding of the fundamentals of the structure in crystals of nanometric size. It is well established that nanocrystals can take a variety of shapes which often appear in competition in the same sample. ${ }^{1}$ These shapes may include icosahedra, decahedra, truncated octahedra and triangular platelets, among others. The appearance of these geometries is somewhat surprising since it is well known that, if we start to construct these nanocrystals starting from FCC tetrahedral units, we will end up with non-physical situations. For instance, a decahedron is made of five tetrahedral units in a cyclic twin sequence; this fivefold structure however will not fill the space and an angular gap

${ }^{a}$ Department Of Chemical Engineering, University Of Texas at Austin, Austin, TX, 78712, USA

${ }^{b}$ Facultad de Ciencias Físico-Matemáticas Universidad Autónoma de Nuevo León, San Nicolás De los Garza N. L., 66450, Mexico of $7.35^{\circ}$ will result. ${ }^{2}$ This situation will generate internal strains on the nanoparticle in order to close the gap and, as a result, an increase of the lattice parameter from the center to the surface of the particle will be observed. Despite the strains, the modification of the lattice makes this kind of shape stable at this size level. ${ }^{3}$ However, as the nanocrystals grow larger than about $5 \mathrm{~nm}$, the internal strain also becomes larger and new defects will be introduced in the structure in order to close the angular gap. This is particularly true when the shape is kinetically trapped, i.e., when the particle gets trapped on a local minimum of the energy landscape during the synthesis process, thermodynamically far from the

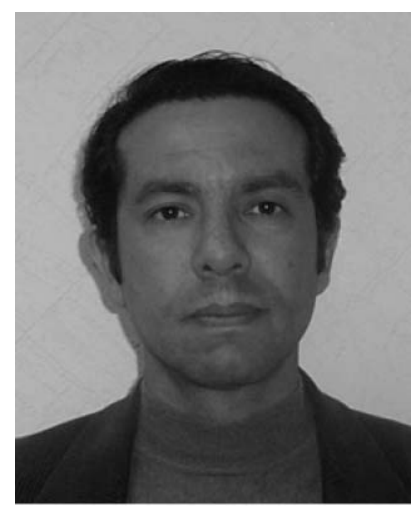

Sergio Mejía-Rosales

Sergio Mejía-Rosales obtained a PhD degree in physics from the Universidad Autónoma de San Luis Potosí, and worked as a postdoctoral fellow at the University of Houston from 2000 to 2002. He currently holds a full-time professor position at the Facultad de Ciencias Físico-Matemáticas, Universidad Autónoma de Nuevo León, where he is responsible for the Molecular Design Laboratory. His interests include research on metallic nanostructures by molecular simulations and quantum calculations.

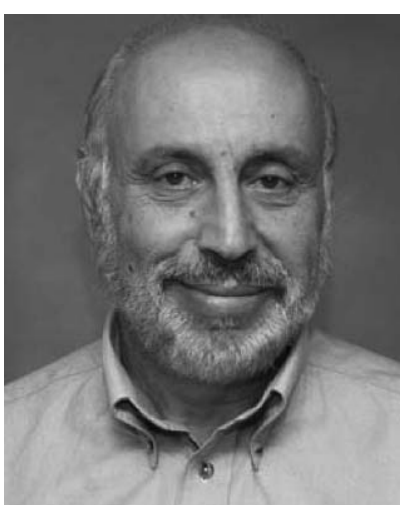

Miguel José-Yacamán
Miguel José-Yacamán earned his PhD degree in physics of materials at the National University of Mexico (UNAM) in 1973. During his career, he has been a researcher at the Institute of Physics of UNAM, where he was director from 1983 to 1991. He directed the National Nuclear Research Institute in Mexico from 1995 to 2000. He joined the faculty of The University of Texas at Austin in 2001, where he currently directs the International Center for Nanotechnology and Advanced Materials. He has carried out pioneering work on the electron microscopy characterization of crystal structures and the shape of metallic particles of nanometric size, on the growth of

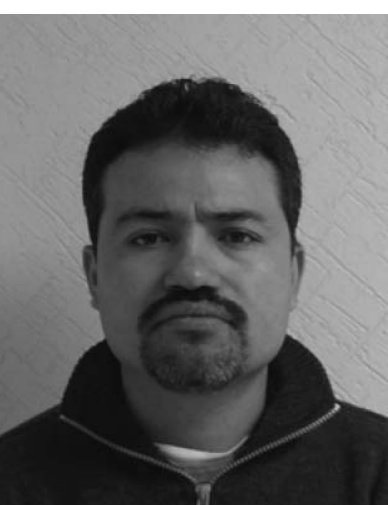

Eduardo Pérez-Tijerina nanostructured materials, and in characterization-supported metal catalysts.

Eduardo Pérez-Tijerina obtained a PhD degree in physics from the Centro de Ciencias de la Materia Condensada, Universidad Nacional Autónoma de México, and currently holds a permanent position as full-time professor at the Facultad de Ciencias Físico-Matemáticas, Universidad Autónoma de Nuevo León (UANL), where he implemented the Nanoscience and Nanotechnology Laboratory, which is the main UANL center devoted to the synthesis, characterization, and technological uses of nanostructures. 

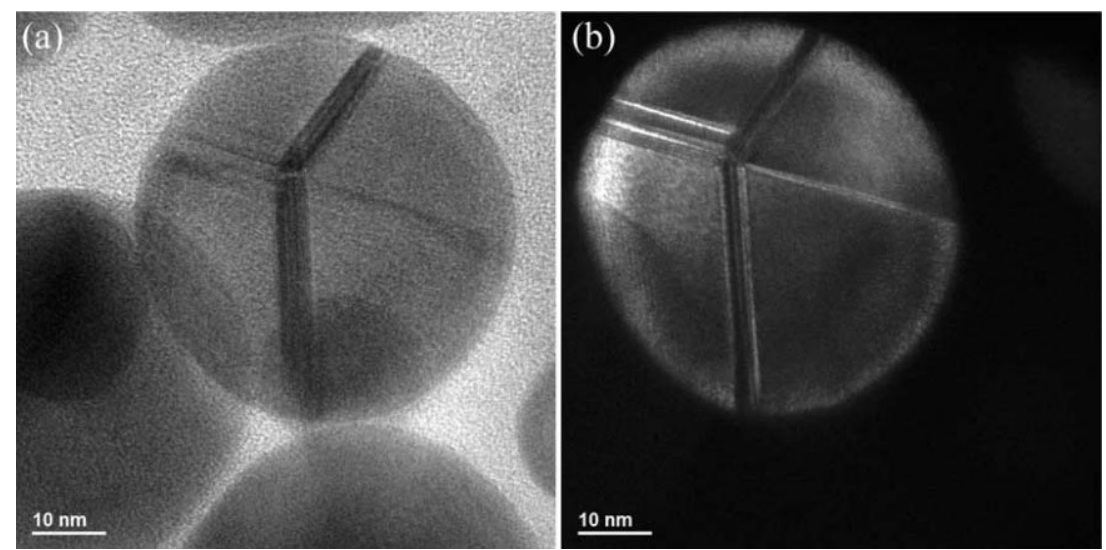

Fig. 1 Images of a twinned Au nanoparticle. Stacking faults can be clearly observed in the interface of twin grains. a) Bright field image; b) dark field image using a (111) reflection.

shape corresponding to the global minimum. An example of this is shown in Fig. 1 for a gold nanoparticle. The particle appears with a quasi-spherical shape in the bright field TEM image, Fig. 1(a). However, the dark field image shown in Fig. 1(b) reveals in a clearer way that there are several defects on the structure at the interface between grains. Here, we can note that the particle is made of several tetrahedral units which have rounded facets. These defects are stacking faults across the interface and nearly parallel to the electron beam direction. Typical fringes produced by the stacking fault can be observed, which can be interpreted as a band of HCP stacked material produced at the interface, such that the regular FCC sequence ABCABCABC becomes an $\mathrm{ABCABCABABABABABCABC}$ sequence. This type of defect was found in results of molecular dynamics calculations by Balleto and Ferrando, ${ }^{4}$ and by Chushak and Bartell. ${ }^{5}$ These authors performed freezing simulations in $\mathrm{Ag}$ and $\mathrm{Au}$ nanoparticles and found that the final structure is formed by sequences of FCC stacking and bands of HCP stacking. This kind of picture is consistent with the available experimental data.

We can now ask the question: what will happen if there are two different metals forming the nanocrystals? Bimetallic nanoparticles have attracted a lot of experimental and theoretical work because of their unique properties. ${ }^{6-9}$ One of the major reasons for the interest in nanoalloy particles is the fact that their chemical and physical properties may be tuned by varying the composition and atomic ordering, as well as by selecting the size of the clusters. The surface structure of the nanoalloys, along with their compositions and segregation properties, are important in determining chemical reactivity, especially catalytic activity. ${ }^{10,11}$ Nanoalloy clusters are also interesting as they may display structures and properties which are distinct from those of the pure elemental clusters. From experimental data using EXAFS and XRD, it is clear that a large number of systems form a core-shell structure. Examples of this are $\mathrm{Pt}-\mathrm{Pd}, \mathrm{Au}-\mathrm{Pd}$ and $\mathrm{Au}-\mathrm{Pt}{ }^{12}$ However, in other cases the evidence suggests the formation of nanoalloys. Examples of the latter are $\mathrm{Cu}-\mathrm{Pd}$ and $\mathrm{Ni}-\mathrm{Pd} .^{13}$ There are also examples of pairs of elements which are immiscible in the bulk phase but which readily mix in finite clusters. ${ }^{14,15}$ In addition, the optical properties of bimetallic nanocrystals may be also important when they are found in the core-shell structure. ${ }^{16,17}$

When we mix two metals to form a nanoparticle there are two situations that can be produced: (i) either the particles form strictly what can be thought of as an alloy, or (ii) a core-shell structure is produced. In the former case, the two atomic species are distributed homogeneously on the particle; in the latter, a core of one of the metals is surrounded by a thin shell of the other metal forming a complex shape. ${ }^{18}$ Usually, the metal with larger size and lower surface energy will be on the outside layer, but the opposite may happen depending on the synthesis conditions. The core-shell for biological applications, especially structure is believed to be a very important factor affecting the electronic properties of the cluster. ${ }^{19}$ However, we should recognize that the former classification represents two extreme cases. In reality, bimetallic particles are more complex. A nanoparticle might have an incomplete core or it may have a eutectic type of structure, where the two metals form separated particles joined in an interface.

Recently, we have performed several molecular dynamics (MD) simulations of the freezing of $\mathrm{Au}$ and $\mathrm{Au}-\mathrm{Pd}$ nanoparticles. The MD simulations were performed in the canonical (NVT) ensemble, emulating a cooling process by making a series of runs starting from a molten state at $1000 \mathrm{~K}$, and decreasing the temperature in intervals of $20 \mathrm{~K}$ until reaching a final equilibrated structure at $300 \mathrm{~K}$. To define the $\mathrm{Au}-\mathrm{Au}, \mathrm{Pd}-\mathrm{Pd}$, and $\mathrm{Au}-\mathrm{Pd}$ interactions, we used the interatomic potential model developed by Raffi-Tabar and Sutton, in a modification of the Sutton and Chen potential, generalized to deal with metal alloys. ${ }^{20}$ Details of the procedure can be found in reference 21 . We simulated the dynamics of particles with different $\mathrm{Au}-\mathrm{Pd}$ concentrations, finding that the final structures that result in the bimetallic case are dramatically different from that of the pure Au particle. Fig. 2 illustrates these differences. In the case of gold particles (see Fig. 2(a)), there is a sharp transition from the liquid state to a well-shaped icosahedral nanoparticle. In the bimetallic particles we found that the behaviour is completely different. In this case there is a transition to a truncated octahedral shape but containing a large number of surface defects, such as kinks, steps, and vacancies, ${ }^{21}$ as can be observed in Fig. 2(b). Therefore, it is clear that the main effect of having two metals is the introduction of a highly defective structure for the nanoparticle.

Even more interesting data can be obtained if we now investigate the local symmetry within the particle. In order to do this we adopted the approach suggested by Chushak and Bartell, ${ }^{5}$ using a local order parameter for every atom, defined as

$$
q_{6}(i)=\left(\frac{4 \pi}{13} \sum_{m=-6}^{6}\left|q_{6 m}(i)\right|^{2}\right)^{1 / 2}
$$




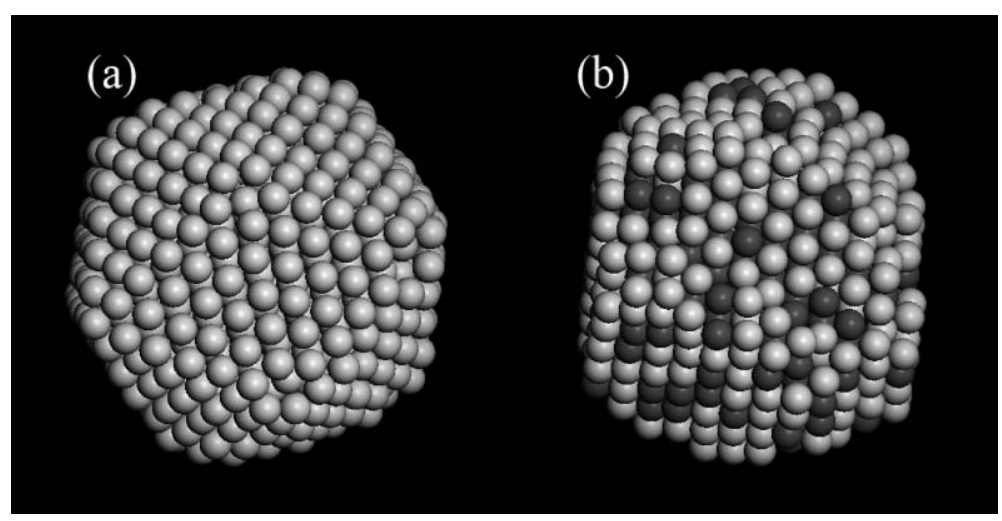

Fig. 2 Final configurations at $300 \mathrm{~K}$ obtained after simulating a freezing process by molecular dynamics. (a) A 923-atom pure Au nanoparticle; (b) a 923-atom AuPd nanoparticle.

where

$$
q_{6 m}(i)=\frac{1}{N_{\text {bonds }}(i)} \sum_{j=1}^{N_{\text {bonds }}(i)} Y_{6 m}\left(r_{i j}\right)
$$

In the last expression, $N_{\text {bonds }}(i)$ is the number of first neighbors surrounding the $i$ th atom on the cluster, and $Y_{6 m}\left(\boldsymbol{r}_{i j}\right)$ are the $6 m$ spherical harmonics for the vector $\boldsymbol{r}_{i j}$ defined by the positions of the $i$ th and $j$ th atoms. Constructed in this way, $q_{6}(i)$ is an appropriate quantitative measure of the kind of environment surrounding the $i$ th atom. Thus, atoms with an FCC-like local structure will have a value of $q_{6}$ close to 0.575 , whereas values around 0.485 will be found in atoms embedded on a HCP local structure. For an isotropic liquid, the average value of $q_{6}$ falls off to zero, which indicates no orientational correlations. Some of the results are shown in Fig. 3, for the same particles as presented in Fig. 2. Here, atoms with an FCC

stacking are plotted in light gray and atoms in a HCP or Ih stacking are inferred from Fig. 3(b), the bimetallic nanoparticle is made of regions with FCC coordination alternated with regions of HCP stacking. Therefore, from these results it can be expected that the electronic properties will be determined not only by the shape but also by the local atomic ordering of the particle. On the other hand, the detailed defect structure will strongly depend on the preparation method: fast cooling will introduce a more defective structure than slow cooling.

An experimental result that seems to support the results of the calculations has been reported by Lucadamo and Medlin, ${ }^{22}$ for the case of gold grain boundaries. Using HRTEM, these authors found intergranular regions with HCP stacking. They found a region in which 5 boundaries intersect. Four of plotted in dark gray. As is immediately

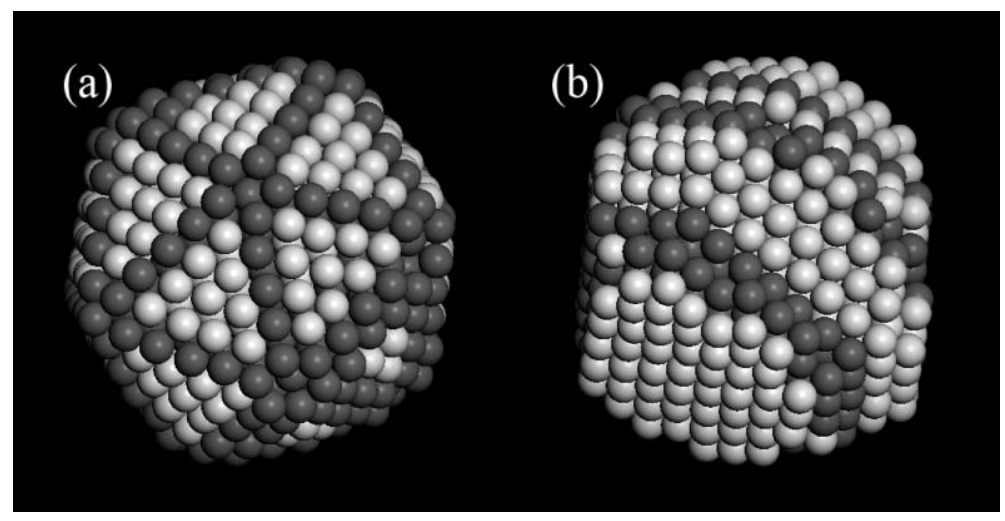

Fig. 3 The same configurations shown in Fig. 2, but here the atoms are colored depending on the value of the local order parameter $q_{6}$. Atoms with an FCC stacking are plotted in light gray, and atoms in a HCP or Ih stacking are plotted in dark gray. (a) A 923-atom pure Au nanoparticle; (b) a 923-atom AuPd nanoparticle. those boundaries are regular (111) twins; however, the fifth boundary shows a dissociation in layers stacked with an ABAB sequence. The authors explain the results in terms of a partial dislocation with a Burgers vector $1 / 6\langle 112\rangle$. This kind of dislocation will shift the lattice to an HCP coordinated site position, which creates a stacking fault. If the array of partial dislocations is periodic, a stacking fault will exit every other plane, and a sequence of the type ABCABC,ABABAB,CBACBA will be produced. A similar result has been reported by Kirkland et al. for the case of CdSe nanoparticles. ${ }^{23}$

There exist different techniques to synthesize bimetallic particles. We prepared bimetallic AuPd nanoparticles by the polyol method, ${ }^{21}$ where hydrogen tetrachloroaurate $\left(\mathrm{HAuCl}_{4}\right)$ trihydrate and $\mathrm{PdCl}_{2}$ were used as precursors. The particles were coated with poly(vinyl pyrrolidone) (PVP), which stabilizes the surface. A drop of the solution with nanoparticles was then dropped on a holey carbon grid for TEM observation. HRTEM was used to study the nanoparticles. A typical image is shown in Fig. 4. The particle shown in the HRTEM image has a dissociated region with HCP ordering at the centre. This experimental evidence seems to support the theoretical calculations, which predict bands of HCP coordinated material intercalated with FCC coordinated material. We found this to be the most representative case. Under our experimental conditions $87 \%$ of the particles showed HCP bands.

It is well established that when dislocations move throughout a lattice, a

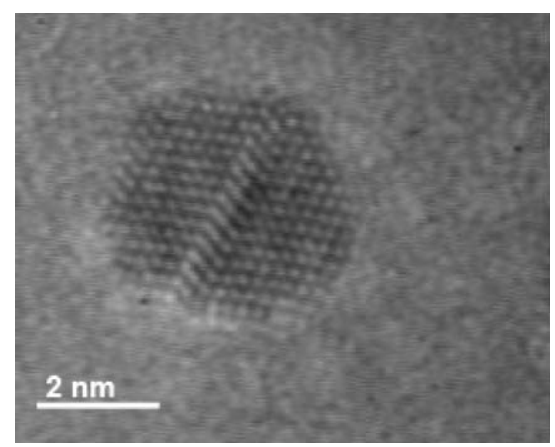

Fig. 4 High resolution TEM image of an $\mathrm{Au}-\mathrm{Pd}$ nanoparticle showing at its center a dissociated region with HCP stacking separated by regions with FCC stacking. 
part of the crystal is displaced with respect to another by a complete lattice vector. However, partial dislocations move one crystal section by less than a complete lattice vector, creating a stacking defect. A nanoparticle cannot sustain a perfect dislocation; however, partial dislocations seem to be produced frequently during the growth process. When there is more than one atomic species present in the particle, differences in mobility of the atoms in a nanoparticle will result, such that partial dislocations are produced during the atomic packing process; this results in a defect structure. For the case of gold nanoparticles, it has been recently shown that an important contribution to electronic structure is due to the low-coordinated sites on the nanoparticle. The number of this type of site will increase by decreasing the particle size. ${ }^{24}$ The number of low coordinated sites will also depend on the shape. Since the presence of defects not only increases the number of low coordinated sites but also controls the particle shape, ${ }^{25}$ we believe that the defect structure of a bimetallic nanoparticle might be a factor, as important as the core-shell morphology, in determining its electronic properties. This factor should be considered in future theoretical and experimental studies of bimetallic nanoparticles.

There is still the issue of the difference of size between the clusters in the MD calculations and the experimental clusters. The former are a few hundred atoms in size while the latter are a few thousand atoms in size. In order to bridge this size gap it is necessary to develop new simulation algorithms that will allow us to work with larger particles at an acceptable computational cost. On the other hand, it is necessary to develop experimental techniques to study smaller nanoparticles. In this sense, the development of a new aberration corrected $\mathrm{TEM}^{26}$ in which sub- $\AA$ resolution can be achieved, opens up new exciting possibilities for comparing calculations and real data.

\section{Acknowledgements}

This work was supported by the Consejo Nacional de Ciencia y Tecnología (CONACyT, grant 43772), the Consejo de Ciencia y Tecnología del Estado de Nuevo León, and the International Center for Nanotechnology and Advanced Materials, The University of Texas at Austin.

\section{References}

1 F. Balleto and R. Ferrando, Rev. Mod. Phys., 2005, 77, 371.

$2 \mathrm{H}$. Hofmeister, Encyclopedia of Nanoscience and Nanotechnology, ed. H. S. Nalwa, American Scientific Publishers, California, USA, 2004, vol. 3, p. 431.

3 T. P. Martin, Phys. Rep., 1996, 273, 199.

4 F. C. Balleto and R. Ferrando, Surf. Sci., 2001, 490, 361.

5 Y. Chushak and L. S. Bartell, Eur. Phys. J., 2001, 16, 43

6 S. Darby, T. Mortimer-Jones, R. L. Johnston and C. Roberts, J. Chem. Phys., 2002, 116, 1536.

7 Large Clusters of Atoms and Molecules, ed. T. P. Martin, Kluwer, Dordrecht, 1996.

8 S. Giorgio, H. Graoui, C. Chapan and C. R. Henry, in Metal Clusters in
Chemistry, ed. P. Braunstein, L. A. Oro, and P. R. Raithby, Wiley-VCH, Weinheim, 1999, vol. 2, p. 1194

9 R. L. Johnston, Philos. Trans. R. Soc. London, Ser. A, 1998, 356, 211.

10 J. A. Rodriguez and W. Goodman, Science, 1992, 257, 897.

11 N. Toshima and T. Yonezawa, New J. Chem., 1998, 22, 1179.

12 N. Toshima, M. Harada, T. Yonezawa, K. Hushihashi and K. Azakura, J. Phys. Chem., 1991, 95, 7448.

13 N. Toshima and Y. Wang, Langmuir, 1994, 10, 4574

14 M. P. Andrews and S. C. O'Brien, J. Phys. Chem., 1992, 96, 8233.

15 A. Christensen, P. Stoltze and J. K. Nørskov, J. Phys.: Condens. Matter, 1995, 7, 1047.

16 K. R. Brown, D. G. Walter and M. J. Natan, Chem. Mater., 2000, 12, 306.

17 Y. Kobayashi, M. A. Correa-Duarte and L. M. Liz-Marzán, Langmuir, 2001, 17(20), 6375 .

18 F. Balleto, C. Morret and R. Ferrando, Phys. Rev. Lett., 2003, 90, 135504.

19 G. Rossi, A. Rapallo, C. Mottet, A. Fortunelli, F. Baletto and R. Ferrando, Phys. Rev. Lett., 2004, 93 , 105503.

20 H. Raffi-Tabar and A. P. Sutton, Philos. Mag. Lett., 1991, 63, 217.

21 S. Mejía-Rosales, C. Fernández-Navarro, E. Pérez-Tijerina, L. F. Allard, D. Bloom and M. José-Yacamán, J. Phys. Chem. B, 2007 , in press.

22 G. Lucadamo and D. L. Medlin, Science, 2003, 300, 1272

23 A. Kirkland, L. Chang and J. Hutchison, JEOL News, 2006, 41, 8

24 N. Lopez, T. V. W. Janssens, B. S. Clausen, Y. Xu, M. Mavrikakis, T. Bligaard and J. K. Nørskov, J. Catal., 2004, 223, 232

25 J. L. Elechiguerra, J. Reyes-Gasga and M. José-Yacamán, J. Mater. Chem., 2006, 16, 3906.

26 M. Lentzen, B. Jahnen, C. L. Jia, A. Thust, K. Tillmann and K. Urban, Ultramicroscopy, 2002, 92, 233. 